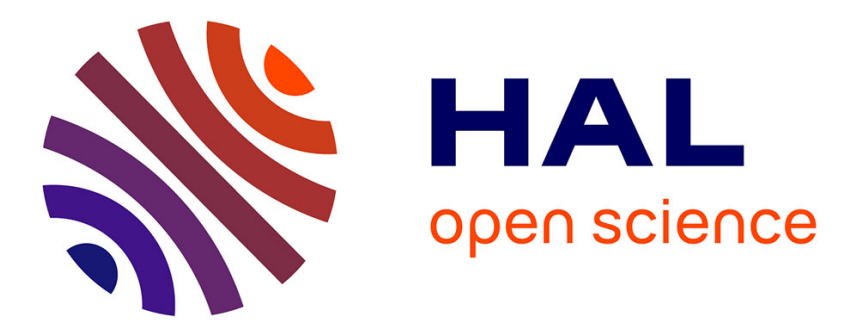

\title{
Weakly Supervised Learning of Deformable Part-Based Models for Object Detection via Region Proposals
}

\author{
Yuxing Tang, Xiaofang Wang, Emmanuel Dellandréa, Liming Chen
}

\section{To cite this version:}

Yuxing Tang, Xiaofang Wang, Emmanuel Dellandréa, Liming Chen. Weakly Supervised Learning of Deformable Part-Based Models for Object Detection via Region Proposals. IEEE Transactions on Multimedia, 2017, 19 (2), pp.393-407. 10.1109/TMM.2016.2614862 . hal-01488575

\section{HAL Id: hal-01488575 https://hal.science/hal-01488575}

Submitted on 16 Mar 2017

HAL is a multi-disciplinary open access archive for the deposit and dissemination of scientific research documents, whether they are published or not. The documents may come from teaching and research institutions in France or abroad, or from public or private research centers.
L'archive ouverte pluridisciplinaire HAL, est destinée au dépôt et à la diffusion de documents scientifiques de niveau recherche, publiés ou non, émanant des établissements d'enseignement et de recherche français ou étrangers, des laboratoires publics ou privés. 


\title{
Weakly Supervised Learning of Deformable Part-Based Models for Object Detection via Region Proposals
}

\author{
Yuxing Tang, Xiaofang Wang, Emmanuel Dellandréa, and Liming Chen Senior IEEE Member
}

\begin{abstract}
Successful deformable part-based models (DPM) for visual object detection relies on training images with fully annotated object bounding boxes. In the context of lack of object level annotations, our goal is to propose a model enhancing the weakly supervised DPM by emphasizing the importance of location and size of the initial class specific root filter. To adaptively select a discriminative set of candidate bounding boxes as this root filter estimate, first, we explore the generic objectness measurement to combine the most salient regions and "good" region proposals. Second, we propose the learning of the latent class label of each candidate window as a binary classification problem, by training category specific classifiers used to coarsely classify a candidate window into either target object or non-target class. Finally, we design a flexible enlarging-and-shrinking postprocessing procedure to modify the output of DPM, which can effectively fit it to the approximative aspect ratio of the object and further improve the final accuracy. Extensive experimental results on the challenging PASCAL Visual Object Class (VOC) 2007 dataset demonstrate that our proposed framework is effective for initialization of the DPM root filter, and it also shows competitive final localization performance with the state-of-the-art weakly supervised object detection methods.
\end{abstract}

Index Terms-Object detection, deformable part-based models, region proposals, weakly supervised learning.

\section{INTRODUCTION}

$\mathbf{O}$ BJECT detection/localization in images is one of the most widely studied problems in computer vision. This task remains challenging mainly due to scale and viewpoint variation, deformation, occlusion, background clutter, intraclass variations and inter-class similarities for the objects in real world images. For most of the existing methods, a fully supervised learning (FSL) approach is adopted [1], [2], [3], [4], where positive training images are manually annotated with bounding boxes encompassing the objects of interest. This manual annotation of object location for large-scale image database is extremely laborious and unreliable thought extremely valuable. However, it is usually much easier to obtain weakly labeled data, where image level labels (e.g., user generated image tags on Internet) are presented. As a result, in this paper, in contrast to the traditional FSL, we are interested in weakly supervised learning (WSL) for object detection, where the exact object locations in positive training examples

Y. Tang, X. Wang, E. Dellandréa and L. Chen are with LIRIS, CNRS UMR 5205, Ecole Centrale de Lyon, F-69134, France. (e-mail: \{yuxing.tang, xiaofang.wang, emmanuel.dellandrea, liming.chen\}@ec-lyon.fr)

This work was partially supported by French Research Agency, Agence Nationale de Recherche (ANR), through the Visen project, under the grant ANR-12-CHRI-0002-04, within the framework of the ERA-Net CHIST-ERA. are not provided, given only the binary labels indicating the presence or absence of the objects of interest.

\section{A. Related work}

In recent years, there has been a substantial amount of work in weakly supervised object detection. From weakly annotated examples, the common practice is to jointly learn an appearance model together with the latent object location. The majority of related work treats WSL for object detection as an MIL (Multiple Instance Learning) [5] problem. In the MIL framework, one has some positive and negative bags. A bag is positive when it has at least one positive instance, while it is negative if all the instances are negative. The objective of MIL is to train a classifier which can correctly classify a test instance as either positive or negative. MIL problems are usually solved by finding a local minimum of non-convex objective function (e.g., MI-SVM [6]). Galleguillos et al. [7] first use the MIL model to recognize and localize objects based on multiple stable segmentations. [8], [9] use variants of MIL to learn object detectors from weakly labeled images and videos. Cinbis et al. [10] use multi-fold training procedure for MIL to avoid rapid convergence to poor local optima. Also in order to get rid of bad local minimum, Song et al. [11] initialize the object locations via a discriminative submodular covering method.

Another main strategy for WSL detection is to utilize a category-independent saliency measure to predict whether a given image region belongs to an object or not. For example, Deselaers et al. [12] propose a fully connected CRF (Conditional Random Field) [13] which aims to select a candidate window with the highest objectness score [14] in each positive training image.

Some work cast the WSL problem as a transfer learning (TL) problem. For example, Shi et al. [15] formulate a TL based on learning to rank, which effectively transfers a model for predicting object location from an auxiliary dataset to a target dataset with completely unrelated object categories. Hoffman et al. [16] propose an algorithm which can learn the difference between the image classifier and the object detector, and transfers this knowledge to classifiers for categories without bounding box annotated data, turning them into detectors. However, for both of these methods, auxiliary object level annotations for part of the dataset are required.

In addition, Pandey et al. [17] modify the fully supervised DPM to a weakly supervised manner without object level 
annotations, which learns structural object detectors based on randomly initialized windows in the positive training images. Shi et al. [18] propose a WSL framework based on Bayesian joint topic modelling which localizes object across different classes concurrently. Recently, Wang et al. [19] propose to learn the latent categories using probabilistic Latent Semantic Analysis (pLSA), and select the target object category by evaluating each latent category's discrimination. Bilen [20] et al. propose to couple a smooth discriminative learning procedure with a convex clustering algorithm, by imposing the similarity among objects of the same class.

\section{B. Motivation and Contribution}

Deformable Part-based Models (DPM) [2] and its variants [21], [22], [23] have been dominant in supervised object detection on challenging PASCAL VOC datasets [24] for a long period. The DPM represents an object with a holistic root filter that approximately covers an entire object and several higher resolution part filters that capture smaller local appearances (parts) of the object. It also characterizes the deformations by links connecting different parts. In the standard (fully supervised) DPM framework, the root filter is initialized with the positive ground-truth object bounding box, and it is allowed to move around in its small neighborhood to maximize the filter score. The locations of object parts are always treated as latent information due to the unavailability of object parts annotations upon most occasions. A latent SVM (LSVM) is adopted to learn the deformation of the objects, which can alternate between fixing latent values (part locations) for positive examples and optimizing its objective function.

Pandey et al. [17] modify the fully supervised DPM to a weakly supervised manner without object level annotations, which treats the location of root filter and part filters full latent, and learns structural object detectors based on the entire image. Its root filter's location is initialized randomly based on a window which has at least $40 \%$ overlap with the positive training image, and its aspect ratio is initialized roughly to the average of the aspect ratios of positive training examples. However, the specific size and location of the initial root filter, as well as their aspect ratio are indicated to have a significant impact on the final localization result [1], [2], [17]. By randomly initialization, the object detector tends to learn spurious models of other classes or background regions, leading to lower accuracy during testing. And to our best knowledge, method for initializing the root filter based on theoretical deduction in weakly supervised DPM, as well as the definition of the aspect ratio of the objects, have not been well studied in [17].

To make up the performance gap between weakly and fully supervised DPM, in this paper, we are motivated to propose a model that enhancing the weakly supervised DPM by emphasizing the importance of location and size of the initial class specific root filter. To be precise, our goal is to discover a reliable initial set of image windows that are probably going to contain the target objects in the positive training images with only category level annotations, so as to represent the object instances. Hence, our WSL framework incorporates adaptive window selection from class independent object proposals and training of deformable part-based models. In particular, we explore the "objectness" approaches [14], [25], which generate class independent object proposals with corresponding scores indicate their probabilities of being object instances, then we adaptively select a reliable set of windows from the derived object proposals for each image as initialization, by fusing visual saliency and "objectness" scores. Two different initialization schemes are developed: single region and multiple regions initilization. The former tends to select one relative larger bounding box which may contain the most salient part in the image, while the latter is much more generalized, which selects a small number of object estimations that can also capture smaller and scattered objects. For multiple regions initialization, the labels of the regions are latent information. We learn the latent class label by framing it as a classification problem, which tries to coarsely classify each region into target object class or nontarget class by some class specific classifiers. The generated object estimations are treated as the initial root filter estimates for training DPM detector.

The main contributions in this work are four-fold:

1) We propose a selection model based on generic "objectness" and visual saliency to adaptively select a discriminative set of candidate windows which tend to represent the object instances in the image.

2) We frame the learning of the latent class label of each candidate window as a binary classification problem, by training category specific classifiers which tries to coarsely classify a candidate window into either target object or non-target class.

3) We propose to use a flexible enlarging-and-shrinking post-processing procedure to modify the predicted output of DPM detector, which can effectively generate more accurate bounding box by better conserving foreground and cropping out plain background regions, to approximatively fit for the aspect ratio of the object.

4) Extensive experiments are carried out on two subsets and the whole set of the challenging PASCAL VOC 2007 database [24] with different criteria, namely annotation accuracy in terms of correct localization on training set, and detection accuracy in terms of average precision on test set. Experimental results demonstrate that our proposed framework is effective for initialization of DPM root filter, and shows competitive final localization performance with the state-of-the-art weakly supervised object detection methods.

A preliminary version of this work appeared in [26], which fuses the generic "objectness" with deformable part-based models for WSL detection. This paper includes that work but significantly extends it in the following ways. Firstly, we explore a much more generalized model M-WDPM (multiple regions initialization for weakly supervised deformable partbased models) which tries to select multiple regions, and we learn the latent label information of these regions in an effective way. This model shows its superiority in discovering not 


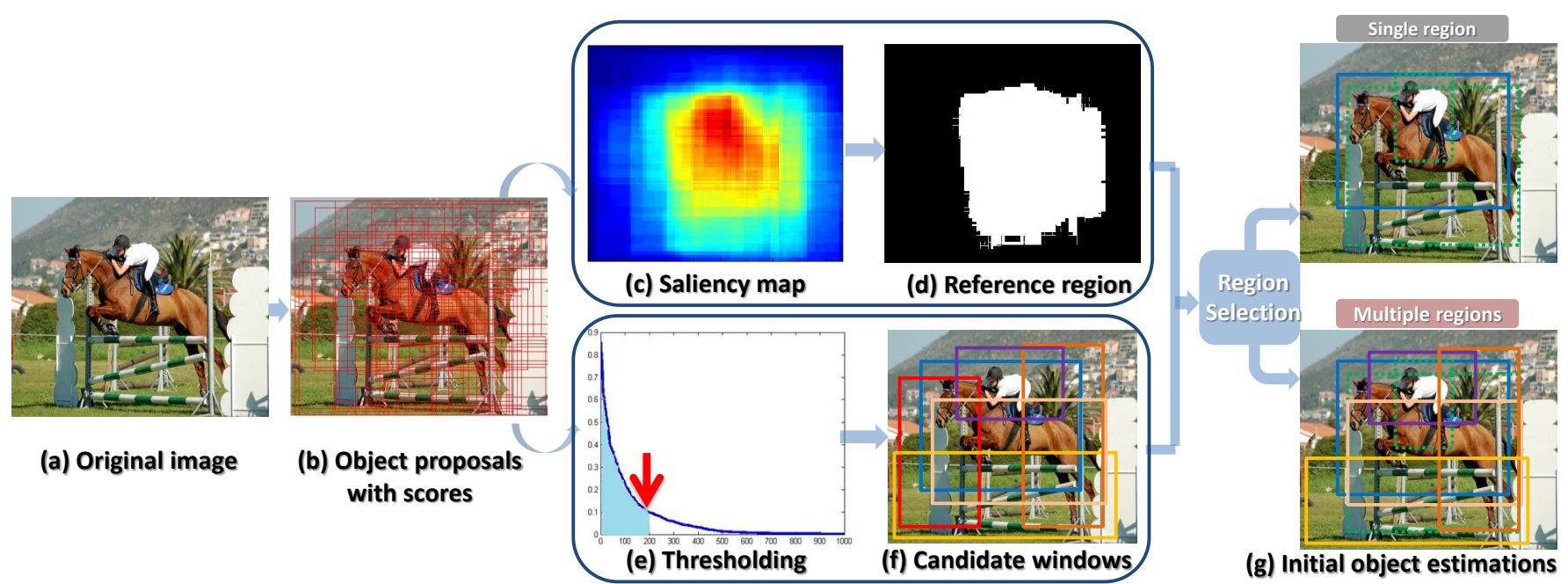

Fig. 1. Illustration of our proposed method to extract the initial object estimations: for an input image (a), object proposals (b) are sampled with corresponding scores to their probability to have object inside by object proposal generating method. (c) is the saliency map derived from (b), and (d) is the reference region obtained by thresholding (c). A coarse set of candidate windows (f) are selected on the sorted proposals (e) by non-maximum suppression (NMS). In the upper image of $(\mathrm{g})$, which indicates the single region selection scheme, the blue window is our initial object estimation obtained by optimizing the overlap between (d) and (f). The bottom image of (g) indicates the multiple regions selection scheme, its color windows with solid lines are multiple finer regions which are assumed to represent the objects in original image. For both images of $(\mathrm{g})$, the green dot line windows are ground-truth bounding boxes for person and horse, respectively.

only salient objects but also smaller and scattered objects to SWDPM (multiple regions initialization for weakly supervised DPM) in [26]. Secondly, we experiment with advanced region proposals generated by Selective Search [25], and we also adopt the latest deep features to represent the image content. Thirdly, we evaluate our framework on the entire PASCAL VOC 2007 dataset, and compare it with state-of-the-arts. We also analyze the types of error that our detection framework inclines to make.

\section{The Organization of the Paper}

The rest of the paper is organized as follows: we present our weakly supervised DPM framework in details in Section II, and in Section III we present our experimental results and the comparison with other methods on PASCAL VOC 2007 datasets. In Section IV, we conclude our work.

\section{Fusing Generic ObJectness And Deformable Part-BAsed Models For Weakly Supervised OBJEct DETECTION}

In this section, we detail our approach of the weakly supervised DPM for object detection. Firstly, we introduce our approach to adaptively select the representative and discriminative regions from the category-independent object proposals. Secondly, we elaborate how to learn the latent class information when multiple regions are selected. Then we briefly describe the weakly supervised learning procedures using the selected regions with DPM and detection rescoring algorith$\mathrm{m}$ for testing. Finally we propose our new post-processing method to further refine the predicted object bounding box obtained by weak DPM detector, so as to cover the object more precisely.

\section{A. Object Estimations: Initialization}

In the weakly supervised DPM training procedure, a good initialization of the root filter is crucial. Hence, our goal is to discover a reliable initial set of image windows that are probably going to contain the target objects in the positive training images with only category level annotations, in order to represent the object instances.

1) Region extraction: Two general approaches have been proposed for generating class-independent object proposals in recent years: window scoring methods such as Objectness [14], BING [27], EdgeBoxes [28] and grouping methods such as Selective Search [25], Constrained Parametric Min-Cuts (CPMC) [29], Multiscale Combinatorial Grouping (MCG) [30]). We use Selective Search since it has been used as the proposal generating method by state-of-the-art supervised RCNN detector [4]. We also report results using objectness method [14] to make comparison with prior detection work [14], [26].

Given an input image $I$ (shown in Fig.1(a)), we first select top $n$ scored windows $W=\left\{w_{1}, w_{2}, \ldots, w_{n}\right\}$ and corresponding scores, denoted as $S=\left\{s_{1}, s_{2}, \ldots, s_{n}\right\}$, which indicate the probabilities to cover objects within them, generated by Selective Search (shown in Fig.1 (b)). To balance a high recall (i.e., covering more objects) and computation efficiency (i.e., small number of region proposals), we set $n=\min (1000, N)$ according to [31], where $N$ is the number of proposals generated by Selective Search.

Based on the fact that the region proposal method is designed to capture all possible objects within an image, we assume that it has the reliability for providing a set of good candidate windows $W^{*} \subseteq W$ which covers the object of interest. However, the windows with the higher scores are not always the effective choices [15], which usually encompass other noisy background, or locate poorly on object targets (e.g., they 
may cover only the object parts). To extract a reliable set of object estimations from the pool of $n$ windows, we design a recursive selection scheme shown in Fig.1 (c)-(g).

2) Salient reference region: For weakly supervised learning, it is obvious the initialization of DPM root filter is significant. It will hurt the detector gravely if it shoots on the background region. Consequently, starting from visually meaningful regions (foreground objects) is imperatively necessary. Inspired by the success of visual saliency applied in salient object recognition, we compute the reference region $R$ (shown in Fig.1 (d)) by thresholding and merging the saliency map (or heat map) $M$ (shown in Fig.1 (c)). The value of saliency map $M$ at pixel $I(i, j)$ is obtained by summing up the scores of the windows that cover this pixel:

$$
M(i, j)=\sum_{k=1}^{n} M_{k}(i, j)
$$

where,

$$
M_{k}(i, j)= \begin{cases}s_{k}, & \text { if } I(i, j) \in w_{k}, \forall w_{k} \in W, \\ 0, & \text { otherwise. }\end{cases}
$$

The reference region $R$ can be one connected (continuous) region or several discrete regions in the image according to the score range and threshold value.

3) Coarse candidate windows pool: It is known that the score given by Selective Search (i.e., objectness score) corresponds with the probability to have target object inside its window to some extent. To take advantage of this auxiliary information, we concurrently select 200 out of $n$ windows that with higher scores as candidates, according to the histogram of $n$ scored windows (shown in Fig.1(e)). In order to avoid near duplicate candidate windows, we further perform nonmaximum suppression (NMS) to get a finer set of candidates. Contrary to the common practice, which starts the suppression procedure from highest scored window, we randomly choose one, because we observed that the window with the highest score is not necessarily the best. Fig. 1 (f) illustrates the derived smaller set of $l$ confident candidates $\hat{W}=\left\{\hat{w}_{1}, \hat{w}_{2}, \ldots, \hat{w}_{l}\right\}$, and their corresponding scores denoted as $\hat{S}=\left\{\hat{s}_{1}, \hat{s}_{2}, \ldots, \hat{s}_{l}\right\}$.

4) Object invariant estimations: Given the reference region $R$ which implies the most salient region (or regions) within an image, and confident candidate windows $\hat{W}$ with scores $\hat{S}$, the overlap between them provides valuable information to find the locations of target objects. We will propose two different schemes to fuse the salient region(s) with the extracted candidate windows.

a) Single region initialization: In [17], the root filter of the DPM is randomly initialized from a single window which covers at least $40 \%$ overlap with the original image. To demonstrate our selection scheme is superior than the randomly chosen window, we also filter out only one single window $w^{*}$ from the candidates pool $\hat{W}$. Intuitively, we expect this window estimation to cover as much as the salient reference region $R$ and to have a relative higher objectness score as well. Hence forth, the estimation of the initial object bounding box with objectness score $\left(w^{*}, s^{*}\right)$ (Fig.1(g), upper image) can be determined by optimizing the following function:

$$
\left(w^{*}, s^{*}\right)=\underset{\hat{w}_{i} \in \hat{W}, \hat{s}_{i} \in \hat{S}}{\arg \max }\left[\alpha \hat{s}_{i}+(1-\alpha) \frac{\operatorname{area}\left(R \cap \hat{w}_{i}\right)}{\operatorname{area}\left(R \cup \hat{w}_{i}\right)}\right], \quad i \in[1, l]
$$

where $\alpha$ is a parameter used to control the influence of the objectness score $s_{i}$. In practice, $\alpha=0.2$, was selected by a grid search over $\{0.1,0.2,0.3,0.4\}$ on a validation set, for the purpose of emphasizing the priority of the intersection over union (IoU) overlap between the candidate window and merged salient reference region.

The single region initialization scheme prefer to select a relative large region which may contain the most salient part in the image. It can produce good DPM object detectors in a weakly supervised manner, when very few objects gathering together in an image. For example, by adopting the single region scheme, the blue window in Fig.1(g) upper image, is used as a positive training example (i.e. DPM root filter initialization) for both horse and person category.

b) Multiple regions initialization: In fact, multiple objects (e.g., 2.5 objects in average for PASCAL VOC2007 trainval dataset) can be scattered anywhere in an image. We can therefore further improve DPM detectors by providing more object estimations as root filter initiliazations, instead of training the object detectors with a single window for each image. For each image, we are motivated to select a small number of object estimations that can also capture smaller and scattered objects, which can better represent the original image. We adopt the similar criteria as the score function Eq. (3). To alleviate the influence of the area of $R$, we set $\alpha$ to be 0.3 . Instead only selecting the maximal scoring window in Eq. (3), we pick out top $Q$ scored windows $W^{*}$ for each image.

After generating several object estimations from each image, the next step is to approximately identify the class label of each estimation given only the labels of the whole image. For example, in Fig. 1(g) bottom image, the color windows with solid lines are associated with the horse and person labels. However, so far we have no idea which object(s) (or even background) is/are inside each bounding box. We will commit ourselves to solve this problem in the next subsection.

\section{B. Learning Latent Object Classes via Region Classification}

For each positive training image, we have generated $Q$ object invariant estimations with the multiple regions initialization scheme. Consider an object category, e.g., horse, which has $P$ positive training images, we can totally obtain $z=P * Q$ object estimations. Obviously, some of these object estimations come from other categories (e.g., person, sheep, object parts or the background regions as well), where the class labels are latent information. In this paper, we frame the latent class learning problem as a classification problem by coarsely classifying these object estimations into either target object category or non-target category (i.e., other classes, object parts or background).

1) Region representation: We use the deep convolutional neural networks ( $\mathrm{CNN})$ features to represent the regions (object estimations). Firstly, we pre-train an eight-layer (five convolutional layers and three fully-connected layers) Alex-Net 

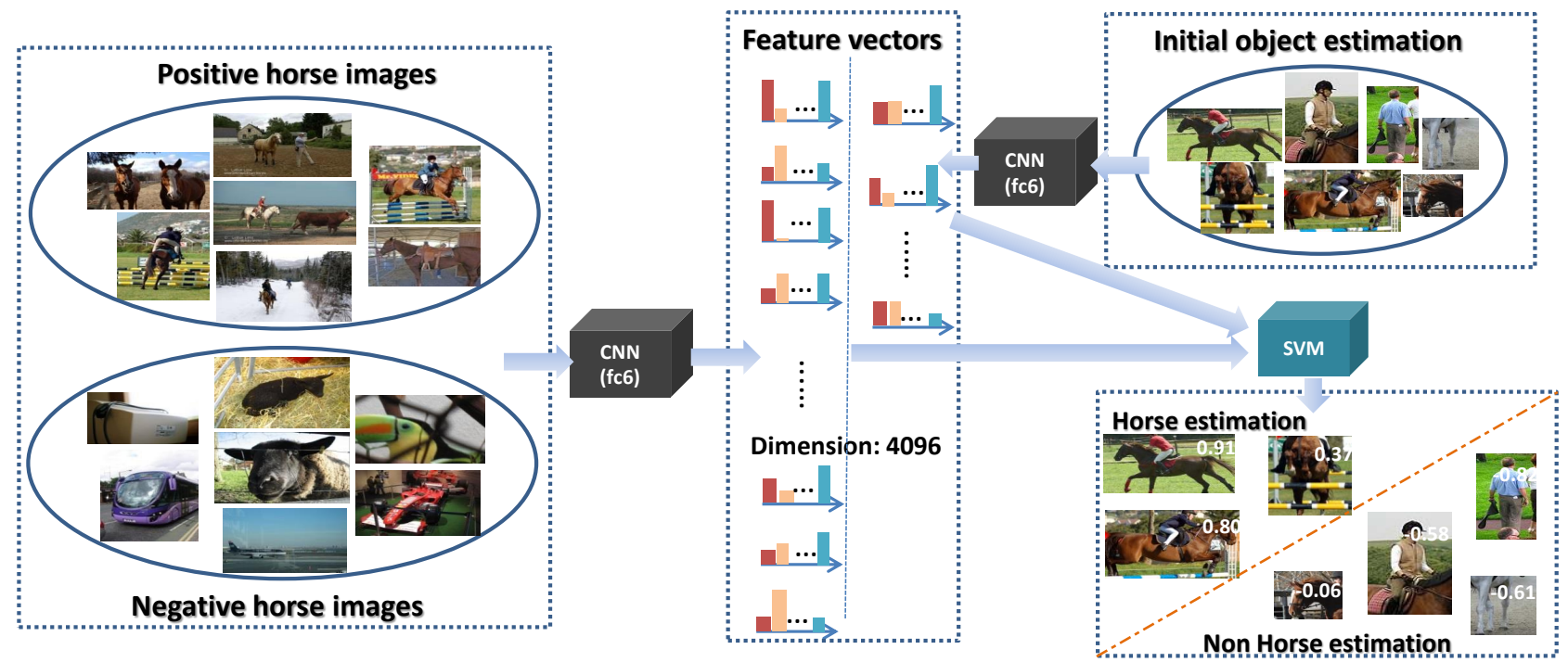

Fig. 2. Illustration of our latent class learning framework for the horse category. For each object category, we train a linear SVM classifier with the CNN features (output of CNN's $f c 6$ layer). Object estimations from the positive training images of this category are scored by its SVM. We select the regions with higher scores by thresholding as the representative objects of this category (horse vs. non horse for this example).

[32] CNN with caffe implementation [33] on the ImageNet Large Scale Visual Recognition Challenge (ILSVRC) 2012 classification dataset [34], which contains 1.2 million images of 1000 categories. Then we warp each region into a required fixed pixel size of $227 \times 227$, and subtract it with the mean RGB image, and forward propogate it through the network. Finally, we take the output of the fist fully-connected layer (i.e., $f c 6$ layer) to represent the input region. The output of $f c 6$ layer is a 4096-dimensional feature vector. This feature extraction process is similar to R-CNN [4], but it is worth noticing that we do not fine-tune the pre-trained CNN on target dataset, because the object level annotations are assumed not to be available in the weakly annotated data. And we do not pad the region with additional image context around it, as our region estimation is already expected to have a significant coverage of the context information due to our selection schemes in Section II-A3.

2) Region classification: Consider training a horse detector. For all the $P$ positive training images in the horse category, we generate $z$ object invariant estimations. Intuitively, only part of these $z$ regions contain the target horse object, others may have person, sheep, dog or even background. We learn the latent categories in these regions via region classification.

We first train a horse linear SVM [35] classifier using the images labeled with horse as positive training examples and the ones without horses as negative examples. We compute the similar 4096-dimensional CNN feature vector as in Section II-B1 on whole images. We then run the trained horse classifier on the $z$ object invariant estimations in the positive training images. By thresholding the SVM scores, finally we obtain a subset $z^{\prime}$ regions from $z$ estimations $\left(z^{\prime}<z\right)$. These $z^{\prime}$ regions are assumed to represent the target horse category, which can be treated as positive training examples of the horse detector.

Suppose we have $K$ categories we want to detect. We train one binary SVM classifier on positive and negative images of each category, and run these $K$ classifiers on their corresponding object estimations. We select high scoring regions for each target category so as to represent the objects of interest. Fig. 2 shows the latent class learning framework using SVM classification on the horse category.

Note that the above latent class learning process is only applied to multiple regions initialization, since for single region initialization, the unique generated window is used to initialize the DPM root filter for any categories appeared in the image.

\section{Weakly Supervised DPM Training and Testing Details}

We design two different kinds of deformable part based models for weakly supervised object detection according to different initialization schemes in Section II-A.

1) Single region initialization for weak DPM (S-WDPM) detection: Similarly to [2], each root filter hypothesis in a positive training image is initialized with the corresponding derived bounding box from the single region initialization scheme. The size and aspect ratio of the DPM root filter are decided by the average size and aspect ratio of the object estimation boxes (ground-truth bounding box and aspect ratio are used in [2]). The root filter hypothesis is allowed to move around in a small neighborhood to maximize the filter score to compensate for imprecise bounding box estimation from Section II-A4a. As in [17], we represent an image by a multiscale HOG feature pyramid [1] of 16 levels. For this DPM model, we use only a single component, since the multiple components is used for detecting objects with different views. We set the number of parts in this DPM to 8 as [2]). And for negative training examples, we use random negatives from other object categories. For testing, sliding window approach is adopted. This single region initialized weakly supervised DPM detection model is denoted as $S$ WDPM. We refer the reader to [2] for more details concerning 
the DPM training and detection procedures.

2) Multiple regions initialization for weak DPM (MWDPM) detection: For the M-WDPM (multiple regions initialized weakly supervised DPM), we make it much "deeper" with the DeepPyramid feature [36], for the reason that the HOG feature is suboptimal compared to deep features computed by CNN [3], [37], [4], [38], [19]. The feature map is computed by the fifth convolutional layer (conv5), which has 256 feature channels. We represent each image (or region) with a feature pyramid of 7 levels (v.s 16 levels for HOG pyramid). For training, the selected object estimations from Section II-B2 are treated as positive training examples, and the random windows from negative images are defined as negative examples. We use a DPM with 3 components and 8 parts per component according to [36]. The training and testing procedures are similar to S-WDPM above, but we add a simple bounding box rescoring stage with the help of a frontto-end CNN padded with a softmax classifier.

The rescoring function is defined as:

$$
s_{\text {det }}^{i}=\kappa s_{M-W D P M}^{i}+(1-\kappa) s_{c l s}^{i}, \quad i \in[1, K]
$$

where, $0 \leqslant s_{M-W D P M}^{i} \leqslant 1$ is the normalized DPM detection score on a sub-window of the $i-t h$ detector, and $0 \leqslant s_{c l s}^{i} \leqslant 1$ is the softmax classification score of the corresponding $i-t h$ category on this sub-window. $\kappa$ is a hyperparameter used to levarage the two scores, which ranges from 0.6 to 0.9 . The final predicted windows are obtained by thresholding the $S_{d e t}^{i}$ in Eq. (4).

In order to train this front-to-end CNN classifier described above, we fine-tune the pre-trained CNN with image level annotations on our training data. We implement it by removing the last 1000-way softmax layer while keeping all the other parameters and adding a new randomly initialized $K$-way softmax classification layer, and we then fine-tune the entire network based on the image-level labels.

In [2], contextual information is exploited to rescore the bounding boxes. However, it needs object-level annotations to extract the contextual information. Our detection rescoring method does not require the object level annotations, and it leads to a remarkable improvement in the average precision on several classes in the PASCAL VOC 2007 datasets (see Section III-B). An example of our bounding box rescoring procedure is shown in Fig. 3.

\section{Bounding Box Post-processing}

In many cases, the bounding boxes generated by DPM detectors are too large (resp. small) when detecting very small (resp. large) objects due to the restrictions of the size of the root filter and the scale of the feature pyramid. To improve the localization and to obtain a more precise prediction of the bounding box aspect ratio, we post-process each bounding box by enlarging or shrinking ( $E S$ post-processing) it to cover the object as much as possible. This is done using an improved version of the method proposed in [39] which measures the amount of area that the edge energy occupies. In brief, we first augment the original bounding box $w=\left(x_{\min }, y_{\min }, x_{\max }, y_{\max }\right)$ to $120 \%$ of the original width and height (i.e., $144 \%$ in total

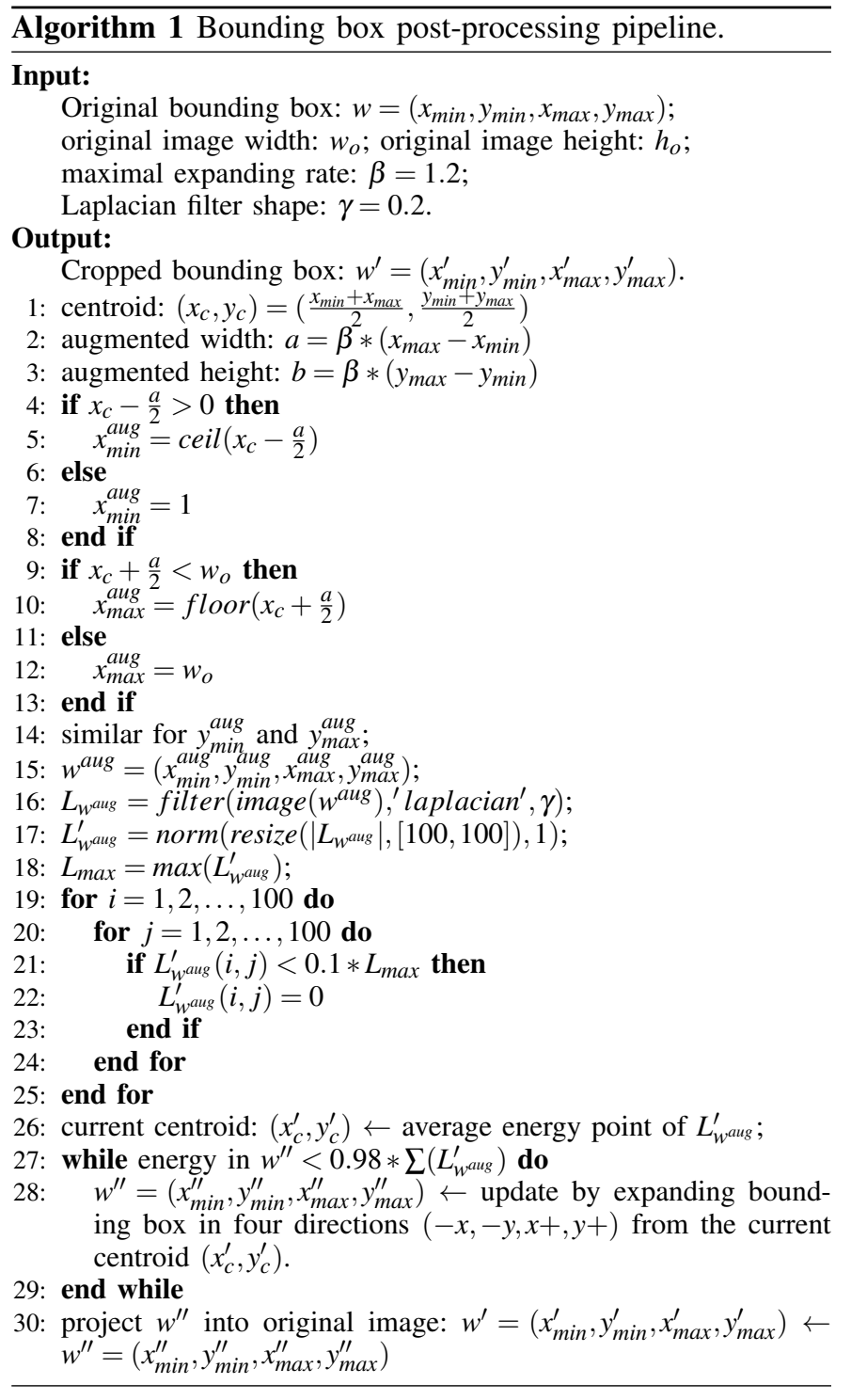

area, denoted as $w^{\text {aug }}=\left(x_{\text {min }}^{\text {aug }}, y_{\text {min }}^{\text {aug }}, x_{\text {max }}^{\text {aug }}, y_{\text {max }}^{\text {aug }}\right)$. Expanding from the centroid if applicable. Otherwise, stop when reaching the border of the image.), and calculate the absolute values of the gradients $L_{w^{a u g}}$ by applying a $3 \times 3$ Laplacian filter with $\gamma=0.2$ over the augmented bounding box. To easily calculate the edge spatial distribution, we then resize the gradient magnitude image size to $100 \times 100$ and normalize the image sum to 1 , i.e., $L_{w^{a}}^{\prime}$.ug. And we set the values which are less than $10 \%$ of the maximum $L_{\max }$ to 0 . Finally, we expand the bounding box in four directions from the current centroid $\left(x_{c}^{\prime}, y_{c}^{\prime}\right)$ and stop until it contains $98 \%$ of the total gradient magnitude (edge energy) in the augmented box. Detailed algorithm is listed in Algorithm 1.

This post-processing technique is not only able to crop out plain background regions, but also can expand to cover the foreground regions which are not encompassed by the original box. However, the cropping method in [17] is probably to fail with the latter. Fig. 4 shows a few examples of our bounding box post-processing results. It is also worth noticing that this post-processing technique works efficiently for the objects with a unique or plain background, but has limited help for 


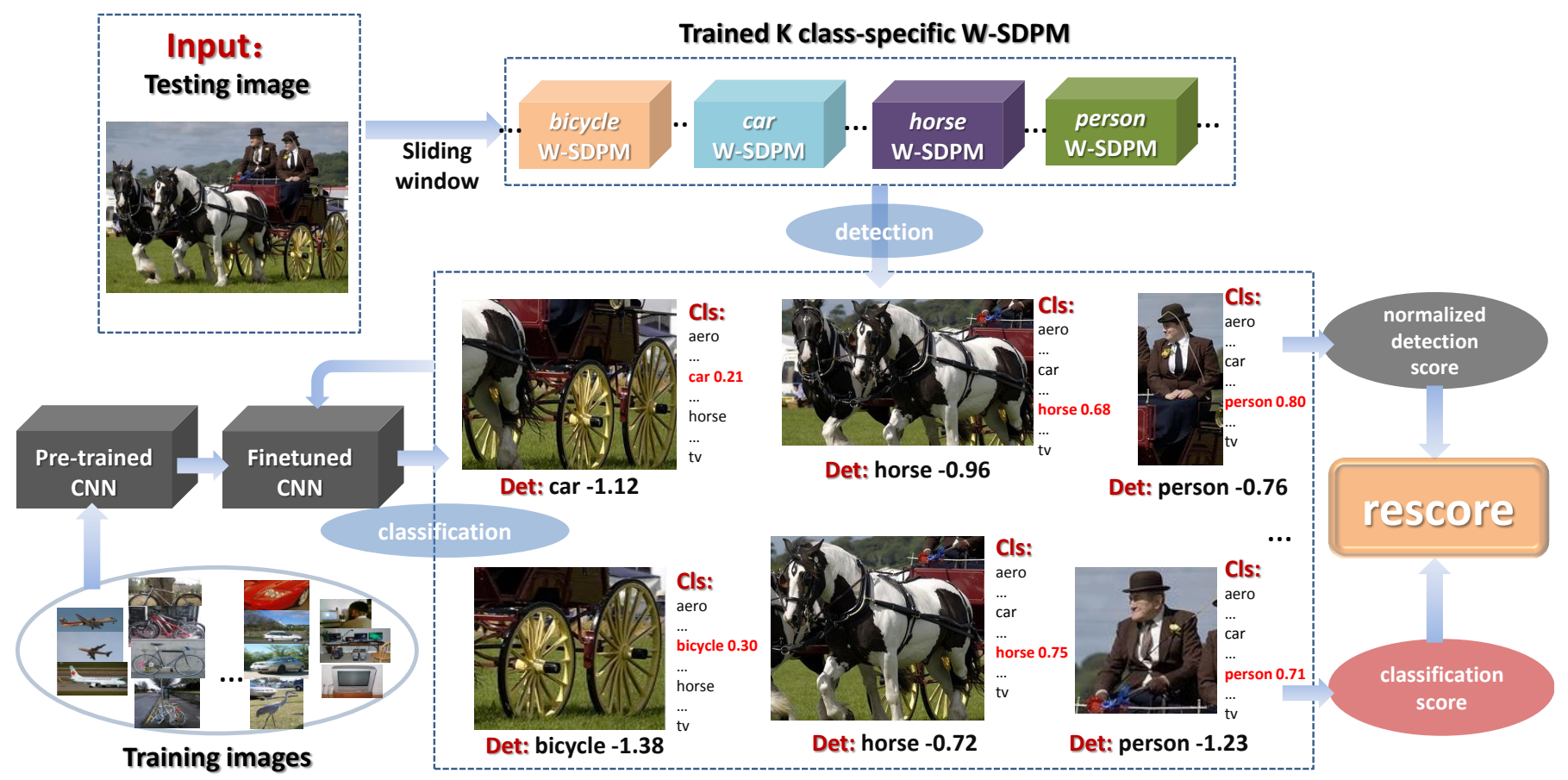

Fig. 3. Illustration of detection rescoring using M-WDPM and CNN softmax classifier. For a testing image, $K$ (number of classes in target dataset) classspecific M-WDPMs are applied on it in sliding window manner. For each sub-window detected by M-WDPM, the normalized detection score is rescored by the softmax classifier of the detected category. In this example, the wrongly detected car and bicycle are finally discarded by the detector after the rescoring stage.
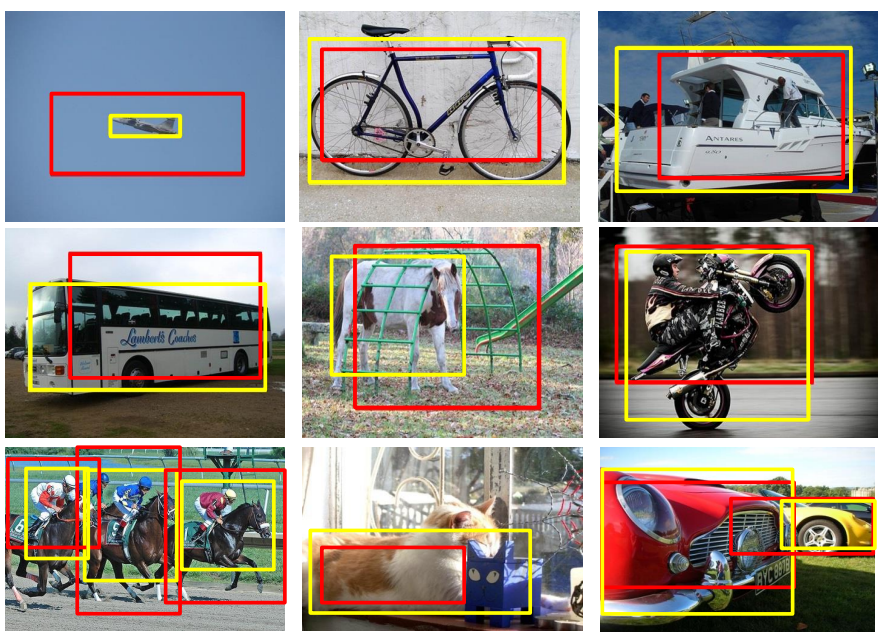

Fig. 4. Examples of bounding box enlarging and shrinking. Boxes before (resp. after) post-processing are shown in red (resp. yellow).

those with cluttered or textured background.

\section{EXPERIMENTAL EVALUATION}

In this section, we present the experimental results of our proposed framework with two different initialization schemes (i.e., S-WDPM using single region initialization and MWDPM using multiple regions initialization) on the challenging PASCAL VOC 2007 dataset [24].

\section{A. Experiments with $S$-WDPM}

1) Datasets: Following the protocol of previous works [17], [12], [40], we evaluate the performance of our proposed SWDPM (single region initialized weak DPM) framework on two subsets from the training and validation set (trainval) of the PASCAL VOC 2007 dataset (VOC07)[24]: VOC07-6×2 and VOC07-14. The VOC07-6×2 subset contains 6 classes (aeroplane, bicycle, boat, bus, horse and motorbike) with Left and Right views (aspects) of each class, resulting in a total of 12 separating classes. The VOC07-14 subset (same with PASCAL07-all defined in [17]) consists of 42 class/view combinations covering 14 classes and 5 views (Left, Right, Frontal, Rear and Unspecified). Similar to [17], [12], [40], we remove all the images annotated as difficult or truncated in both training and evaluation steps.

2) Evaluation protocol: To make fair comparisons, we only choose the detection window with highest score per image, although our method can detect multiple instances appeared in the image using sliding window approach. We also report both results for initial and refined localization as [17], [40]. A refined localization is obtained by an iteratively trained DPM detector for one/sevral iteration(s) to refine the initial detection using the previous annotations as ground truth. Performance is evaluated with the percentage of training (train + val) images in which an object is correctly covered by the window (i.e. CorLoc [12]), if the strict PASCAL-overlap criterion is satisfied (intersection-over-union $>0.5$ ).

3) Experimental evaluation: We compare our S-WDPM with Weak DPM [17], Weak objectness [12] and Joint topic model [18]. For the Weak objectness approach [12], the region proposal with the highest "objectnes" score is selected as the 
TABLE I

AVERAGE LOCALIZATION ACCURACY (IN \%) OF OUR S-WDPM (SINGLE REGION INITIALIZED WEAK DPM WITH HOG FEATURES) COMPARED WITH STATE-OF-THE-ART COMPETITORS ON THE TWO VARIATIONS OF THE PASCAL VOC 2007 DATASETS. "CROP” AND "ES" DENOTE THE CROPPING METHOD FROM [17] AND OUR ENLARGING \& SHRINKING POST-PROCESSING. "obj” AND "SS" DENOTE THE OBJECTNESS AND SELECTIVE SEARCH REGION PROPOSAL GENERATING METHOD. " $S$ " AND " $G$ " DENOTE THE SAMPLING AND GAUSSIAN STRATEGY FROM [18].

\begin{tabular}{|c|c|c|c|c|c|c|c|c|c|c|}
\hline & \multicolumn{3}{|c|}{ no post-processing } & \multicolumn{5}{|c|}{ with post-processing } & & \\
\hline & \multirow[t]{2}{*}[17]{} & \multicolumn{2}{|c|}{ S-WDPM } & \multirow[t]{2}{*}{ [17]-crop } & \multicolumn{2}{|c|}{ S-WDPM(crop) } & \multicolumn{2}{|c|}{ S-WDPM(ES) } & \multicolumn{2}{|c|}{$[18]$} \\
\hline & & $o b j$ & $S S$ & & $o b j$ & $S S$ & $o b j$ & $S S$ & $S$ & $G$ \\
\hline Dataset & \multicolumn{10}{|c|}{ VOC07-6×2 } \\
\hline Initialization & 37.22 & 38.74 & 41.52 & 44.62 & 47.85 & 48.40 & 48.59 & 51.01 & 50.8 & 51.5 \\
\hline Refinement 1 & 51.63 & 55.85 & 63.31 & 53.11 & 56.78 & 64.25 & 58.02 & 67.13 & 65.5 & 66.1 \\
\hline Refinement 2 & 56.99 & 59.82 & 一 & 59.31 & 63.31 & - & 63.91 & 一 & - & 一 \\
\hline Refinement 3 & 59.32 & - & - & 61.05 & - & - & - & 一 & - & - \\
\hline Result from [12] & \multicolumn{10}{|c|}{50.00} \\
\hline Dataset & \multicolumn{10}{|c|}{ VOC07-14 } \\
\hline Initialization & 19.98 & 21.73 & 24.87 & 23.00 & 24.20 & 26.30 & 25.12 & 31.84 & 32.2 & 30.5 \\
\hline Refinement 1 & 25.11 & 27.46 & 31.15 & 26.38 & 28.21 & 33.10 & 28.94 & 34.91 & 33.8 & 32.5 \\
\hline Refinement 2 & 27.69 & 28.95 & - & 29.39 & 32.87 & - & 32.82 & 一 & - & 一 \\
\hline Refinement 3 & 28.98 & - & - & 30.31 & - & 一 & - & - & - & 一 \\
\hline Result from [12] & \multicolumn{10}{|c|}{26.00} \\
\hline
\end{tabular}

predicted window. As Table I shows, our method outperforms [12] and our baseline approach [17] on both datasets. Both [17] and our S-WDPM use the same HOG feature pyramid for the DPM. We present our results using two kinds of object proposal generating methods: objeness (obj) and Selective Search $(S S)$. For $o b j$, our average performance of initial detection before post-processing the bounding boxes on the VOC07-6 $\times 2$ and VOC07-14 subsets is $38.74 \%$ and $21.73 \%$ respectively, versus $37.22 \%$ and $19.98 \%$ for [17]. These improvements are due to the initial object estimate of our method described in Section II-A4a, which gives a better initialization of the root filter of DPM detectors. We can also observe that both the postprocessing method of cropping [17] (i.e., S-WDPM(crop) in Table I) and our enlarging-or-shrinking (i.e., S-WDPM(ES)) post-processing method steadily improve the average localization accuracy. In particular, our ES method is superior to the cropping method of [17], as our cropped bounding box is not only able to shrink to crop out the background regions, but also capable of enlarging to cover the whole foreground object resulted by incomplete coverage of the original window. An example is shown in the last row of Fig. 5, where the target object (motorbike) is only partially localized by the initial detector (shown in red rectangles in the middle and right images) for both [17] and our method. However, in the final detection (shown in yellow) after post-processing, our method is able to enlarge the bounding box to approximately include the whole object, while [17] tends to crop out both foreground and background regions.

Additionally, the rows start with "Refinement" in Table I indicate that localization accuracy can benefit from the iterative refinement process. It is worth mentioning that with a better initialization, our models converge to a steady level of performance after one less round of costly re-training (i.e., 2 iterations for $o b j$ vs. 3iterations) than [17], and achieve slightly better results in the mean time.

The detailed comparisons for our S-WDPM using obj with
TABLE II

Class LEVEl LOCALISATION ACCURACY (IN \%) FOR THE VOC07-6×2 DATASET FOR OUR S-WDPM(ES) USING objectness PROPOSALS vs. [17], [12], [40].

\begin{tabular}{l||c|c|c||c|c|c}
\hline \multicolumn{1}{c||}{} & \multicolumn{3}{c||}{ Initialisation } & \multicolumn{3}{c}{ Refined by detector } \\
\cline { 2 - 7 } & ours & {$[17]$} & {$[40]$} & ours & {$[17]$} & {$[12]$} \\
\hline aero left & $\mathbf{6 5 . 1}$ & 55.8 & 39.1 & $\mathbf{6 9 . 7}$ & 65.1 & 58.0 \\
\hline aero right & $\mathbf{6 4 . 1}$ & 61.5 & 50.0 & $\mathbf{8 4 . 6}$ & 82.1 & 59.0 \\
\hline bike left & 31.3 & $\mathbf{3 1 . 3}$ & 28.4 & 85.4 & $\mathbf{8 7 . 5}$ & 46.0 \\
\hline bike right & 42.0 & $\mathbf{4 4 . 0}$ & 30.6 & 54.0 & $\mathbf{6 8 . 0}$ & 40.0 \\
\hline boat left & 9.1 & 4.6 & $\mathbf{1 5 . 1}$ & $\mathbf{1 3 . 6}$ & 2.3 & 9.0 \\
\hline boat right & 9.3 & 9.3 & $\mathbf{2 0 . 7}$ & 14.0 & 7.0 & $\mathbf{1 6 . 0}$ \\
\hline bus left & 23.8 & 23.8 & $\mathbf{3 1 . 0}$ & $\mathbf{4 2 . 9}$ & 28.6 & 38.0 \\
\hline bus right & $\mathbf{6 5 . 2}$ & 52.2 & 35.1 & 69.6 & 47.8 & $\mathbf{7 4 . 0}$ \\
\hline horse left & $\mathbf{6 4 . 6}$ & 60.4 & 48.5 & $\mathbf{8 7 . 5}$ & 83.3 & 58.0 \\
\hline horse right & $\mathbf{7 3 . 9}$ & 67.4 & 45.2 & 76.1 & $\mathbf{8 0 . 4}$ & 52.0 \\
\hline mbike left & $\mathbf{6 4 . 1}$ & 48.7 & 46.3 & 87.2 & $\mathbf{9 2 . 3}$ & 67.0 \\
\hline mbike right & 70.6 & $\mathbf{7 6 . 5}$ & 55.3 & 82.4 & $\mathbf{8 8 . 2}$ & 76.0 \\
\hline average & $\mathbf{4 8 . 6}$ & 44.6 & 37.1 & $\mathbf{6 3 . 9}$ & 61.1 & 50.0 \\
\hline
\end{tabular}

the state-of-the-arts on the VOCO7-6 $\times 2$ dataset are listed in Table II. The results show that our method outperforms [17] for most of the categories. Especially, our method achieves the state-of-the-art results in some classes where the target object possesses the most salient regions in that category (e.g., aeroplane, bus, horse). Interestingly, even without refinement process, the accuracy for our method with certain category (e.g., aeroplane left) is superior to the competitors with the time-consuming refinement procedure. Fig. 5 visually compares some of our results with those of [17].

We find that the best detection result using the Selective Search $(63.31 \%)$ is $3.49 \%$ better than the objectness $(59.82 \%)$ within the same S-WDPM detection model without postprocessing, and is $3.22 \%$ better $(67.13 \%$ vs. $63.91 \%)$ with post-processing, on the VOC07-6 62 dataset. This is in accord 
with the conclusion in [31]. Moreover, it achieves comparable or slightly better results than the sophisticated joint topic learning models in [18] with running DPM refinement only once. As shown in Table I, the $S S$ also outperforms $o b j$ on the VOC07-14 dataset. Consequently, we entirely adopt the Selective Search method ('fast' option) for our next experiments.

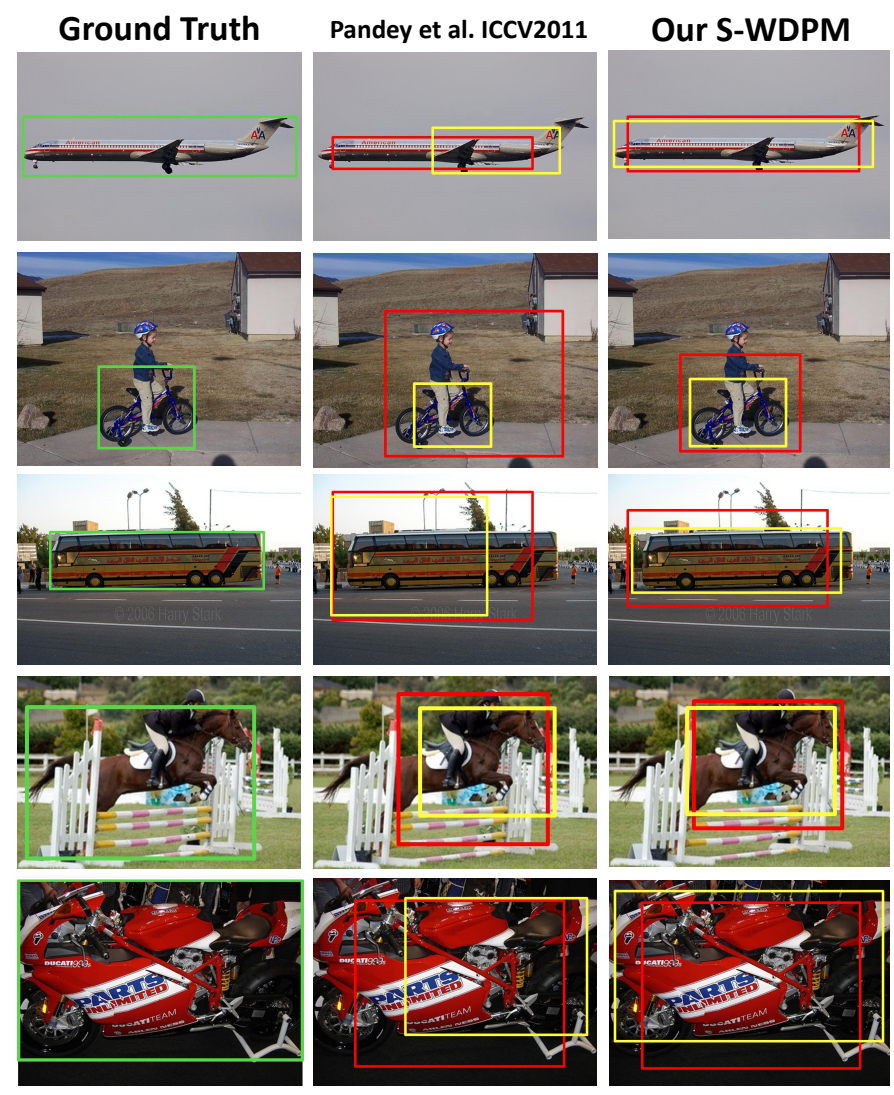

Fig. 5. Examples of localization results of our S-WDPM on PASCAL VOC 2007 images. The left column: ground-truth bounding boxes in green rectangles. The middle and right columns are detection results with [17] and our S-WDPM framework, respectively. Initial detections are shown in red and detections refined by detectors are shown in yellow. Both results are with individual post-processing approach.

\section{B. Experiments with $M$-WDPM}

1) Dataset and settings: We evaluate our generalized model: M-WDPM (multiple regions initialized weak DPM) on the much more challenging dataset: the whole PASCAL VOC 2007 dataset. It contains totally 9963 images of 20 object categories, which is split into training (2501), validation (2510) and test (4952) sets. This dataset is challenging because it has large inter-class similarities, intra-class variances, cluttered backgrounds, and scale changes. We only use the image level category labels for this task. And images labeled as "difficult" ones are discarded as common practice in previous studies. For the M-WDPM testing, we only run the DPM once for efficiency, although the iterative detector refinement can steadily improve the final performance. The annotation accuracy on the trainval (training + validation) set and average precision (AP) for detection on the test set are reported.
For the DeepPyramid feature extraction, we use a single NVIDIA GeForce GTX 780 GPU with 3GB memory. And we reduce the resized image resolution from $1713 \times 1713$ in [36] to $1505 \times 1505$ to avoid running out of memory.

2) Parameter selection: As discussed in Section II-A4b, we can generate $Q$ region estimations for each image. $Q$ is a parameter which impacts the quality of the positive training examples. If it is too large, there would be an enormous number of noisy samples for latent class learning. If it is set to be very small, the instances in the original image would not able to be comprehensively represented. Therefore, we experimentally vary $Q=\{3,5,10,15,20,30\}$ to see which one performs best on the PASCAL VOC 2007 validation set. We implement this by directly measuring the average annotation accuracy for all the classes, on the generated bounding boxes ( $Q$ per image) with the Pascal-overlap criterion. Fig. 6 shows the annotation accuracy for different $Q$. We find that $Q=10$ obtains the best result (34.5\% average accuracy). When it is very small (e.g., 3), the performance drops dramatically to $27.0 \%$. This is because some of the "good" region proposals are not selected due to very small $Q$, while some selected "bad" regions may harm the model. When it goes up from 10 to 30 , the performance declines gradually. One explanation for this might be that many object parts or background regions would be included when $Q$ is large. Hence, we set $Q=10$ in all of our experiments. Fig. 7 shows three example images and their 10 selected regions. The $\kappa$ in Eq. (4) which leverages the classification and detection scores is set to 0.7 according to cross-validation on a subset of the validation data.

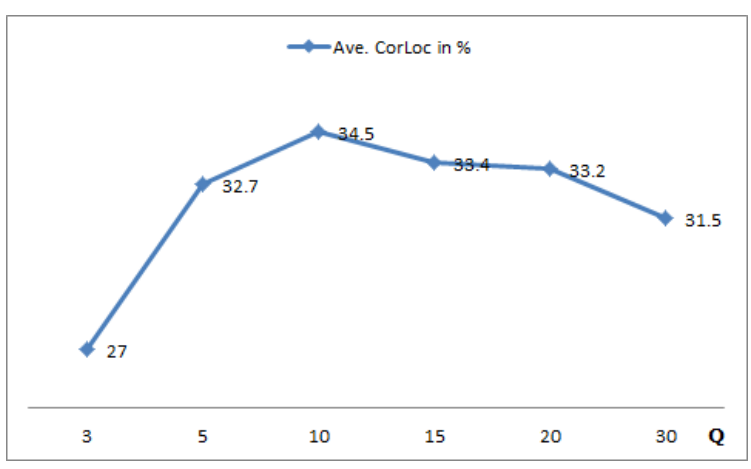

Fig. 6. The impact of parameter $\mathrm{Q}$ (number of selected regions for each image in multiple regions initialization scheme). The average annotation accuracy on PASCAL VOC 2007 validation is evaluated with different $Q$.

3) Annotation evaluation: We evaluate the same CorLoc [12] as in Section III-A2 on the PASCAL VOC 2007 trainval set. Table III reports our experimental results compared with the state-of-the-art WSL methods for object detection.

For our M-WDPM-HOG baseline which computes the HOG features and does not make use of auxiliary training data from ILSVRC 2012 classification task [34] as [20], [19], it outperforms most of the previous works [8], [41], [9], [40], [15], [18] (ours: $36.8 \%$ vs. best of the previous works (Joint topic): $36.2 \%$ ). Our M-WDPM-HOG shows modest improvement in most of the classes, which proves that our multiple regions initialization method has very discriminative power to select the "good" regions in the original image for 
TABLE III

COMPARISONS OF WEAKLY SUPERVISED OBJECT DETECTORS ON PASCAL VOC 2007 TRAINVAL SET IN TERMS OF CORRECT LOCALIZATION (CORLOC [12], IN \%) ON POSITIVE TRAINING IMAGES. († INDICATES METHODS USING AUXILIARY TRAINING DATA FROM ILSVRC 2012.)

\begin{tabular}{|c|c|c|c|c|c|c|c|c|c|c|c|}
\hline method / class & aero & bike & bird & boat & bottle & bus & car & cat & chair & cow & \\
\hline our M-WDPM-HOG & 67.6 & 51.7 & 32.2 & 20.1 & 14.7 & 41.6 & 58.8 & 57.1 & 9.0 & 41.9 & \\
\hline our M-WDPM-deep ${ }^{\dagger}$ & 71.2 & 58.1 & 37.0 & 22.4 & 17.1 & 44.6 & 62.4 & 60.2 & 17.3 & 48.8 & \\
\hline our M-WDPM-rescore ${ }^{\dagger}$ & 82.1 & 55.1 & 42.8 & 35.3 & 14.8 & 57.9 & 66.2 & 69.8 & 17.5 & 51.6 & \\
\hline Joint Learning [8] & 30.7 & 16.5 & 23.0 & 14.9 & 4.9 & 29.6 & 26.5 & 35.3 & 7.2 & 23.4 & \\
\hline MI-SVM [41] & 37.8 & 17.7 & 26.7 & 13.8 & 4.9 & 34.4 & 33.7 & 46.6 & 5.4 & 29.8 & \\
\hline Model Drift [9] & 42.4 & 46.5 & 18.2 & 8.8 & 2.9 & 40.9 & 73.2 & 44.8 & 5.4 & 30.5 & \\
\hline MIL-Negative [40] & 45.8 & 21.8 & 30.9 & 20.4 & 5.3 & 37.6 & 40.8 & 51.6 & 7.0 & 29.8 & \\
\hline Transfer Learning & 54.7 & 22.7 & 33.7 & 24.5 & 4.6 & 33.9 & 42.5 & 57.0 & 7.3 & 39.1 & \\
\hline Joint Topic [18] & 67.3 & 54.4 & 34.3 & 17.8 & 1.3 & 46.6 & 60.7 & 68.9 & 2.5 & 32.4 & \\
\hline $\lg ^{\dagger}[20]$ & 66.4 & 59.3 & 42.7 & 20.4 & 21.3 & 63.4 & $\mathbf{7 4 . 3}$ & 59.6 & 21.1 & 58.2 & \\
\hline LCL-pLSA $^{\dagger}[19]$ & 80.1 & 63.9 & 51.5 & 14.9 & 21.0 & 55.7 & 74.2 & 43.5 & 26.2 & 53.4 & \\
\hline method / class & table & $\operatorname{dog}$ & horse & mbike & person & plant & sheep & sofa & train & tv & mean \\
\hline our M-WDPM-HOG & 13.5 & 37.2 & 45.6 & 57.2 & 18.7 & 19.9 & 36.0 & 22.7 & 43.7 & 46.2 & 36.8 \\
\hline our M-WDPM-deep ${ }^{\dagger}$ & 17.2 & 37.4 & 52.4 & 60.5 & 20.7 & 28.8 & 36.9 & 24.3 & 50.7 & 48.6 & 40.8 \\
\hline our M-WDPM-rescore ${ }^{\dagger}$ & 24.4 & 39.4 & 55.9 & 51.0 & 24.2 & 22.9 & 44.2 & 19.7 & 52.1 & 45.2 & 43.5 \\
\hline Joint Learning [8] & 20.5 & 32.1 & 24.4 & 33.1 & 17.2 & 12.2 & 20.8 & 28.8 & 40.6 & 7.0 & 22.4 \\
\hline MI-S & 14.5 & 32.8 & 34.8 & 41.6 & 19.9 & 11.4 & 25.0 & 23.6 & 45.2 & 8.6 & 25.4 \\
\hline Model Drift [9] & 19.0 & 34.0 & 48.8 & 65.3 & 8.2 & 10.6 & 16.7 & 32.3 & 54.8 & 5.5 & 30.4 \\
\hline MIL-Negative [40] & 27.5 & 41.3 & 41.8 & 47.3 & 24.1 & 12.2 & 28.1 & 32.8 & 48.7 & 9.4 & 30.2 \\
\hline Transfer Learning [15] & 24.1 & 43.3 & 41.3 & 51.5 & 25.3 & 13.3 & 28.0 & 29.5 & 54.6 & 11.8 & 32.1 \\
\hline Joint Topic [18] & 16.2 & 58.9 & 51.5 & 64.6 & 18.2 & 3.1 & 20.9 & 34.7 & 63.4 & 5.9 & 36.2 \\
\hline Convex Clustering $^{\dagger}[20]$ & 14.0 & 38.5 & 49.5 & 60.0 & 19.8 & 39.2 & 41.7 & 30.1 & 50.2 & 44.1 & 43.7 \\
\hline LCL-pLSA $^{\dagger}[19]$ & 16.3 & 56.7 & 58.3 & 69.5 & 14.1 & 38.3 & 58.8 & 47.2 & 49.1 & 60.9 & 48.5 \\
\hline
\end{tabular}
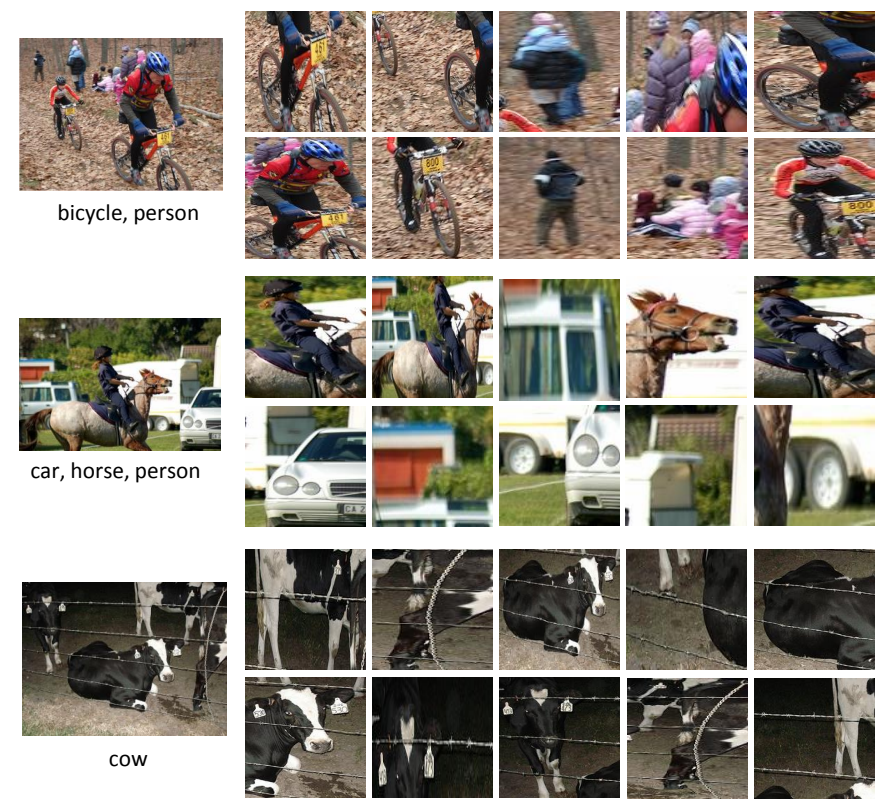

Fig. 7. Three example images and their 10 selected regions (resized to same squared size for regularity).

training the DPM root filters.

It is also observed that, with the help of auxiliary training data and recently popular deep features, the average accuracy of our M-WDPM-Deep model increases by $4 \%$ over the MWDPM-HOG model. And our detection rescoring method (i.e., M-WDPM-rescore) further improves the performance for most of the categories. The average improvement for detection rescoring on all 20 classes is $2.7 \%$ (43.5\% vs. $40.8 \%$ ). Our M-WDPM-rescore method is comparable with the newly invented convex clustering approach [20], but it is worse than the LCL method [19] on average. Though [19] achieves the state-of-the-art performance on many classes, it depends on more sophisticated Super-Vector Coding [44] of the deep CNN features that tragically increases the feature dimensionality. And it fails on some categories such as boat and table. However, our W-SDPM-rescore exhibits steady agreeable performance on all the categories. Especially, our WSDPM-rescore works well on categories where target objects are relatively salient. (e.g., aeroplane, boat, bus, cat and table.) Moreover, it achieves the best results for the classes such as aeroplane, boat, and cat.

4) Detection evaluation: Table IV shows the comparison of our M-WDPM and other methods for object detection on the PASCAL VOC 2007 test set. Our M-WDPM-HOG baseline method achieves an mAP of $22.6 \%$, which outperforms [9] (13.9\%) by a big margin, and is slightly better than [10] $(22.4 \%)$. Both [9] and [10] represent the image windows with SIFT [45] descriptor. [9] uses a Bag-of-Words (BOW) [46] histogram of 2000 dimension, while [10] use Fisher Vectors (FV) encoding [47] to represent the candidate windows. [17] uses the same HOG pyramid features Among these methods that adopt low level visual features, our M-WDPM-HOG works best. Although [11] utilizes powerful deep CNN features 
TABLE IV

COMPARISON OF WEAKLY SUPERVISED OBJECT DETECTORS ON PASCAL VOC 2007 IN TERMS OF AP (AVERAGE PRECISION, IN \%) IN THE TEST SET. ( ${ }^{\dagger}$ SUPERVISED METHODS USING OBJECT LEVEL ANNOTATIONS.)

\begin{tabular}{|c|c|c|c|c|c|c|c|c|c|c|c|}
\hline method / class & aero & bike & bird & boat & bottle & bus & car & cat & chair & cow & \\
\hline our M-WDPM-HOG & 34.1 & 41.5 & 15.2 & 10.0 & 8.8 & 36.5 & 40.8 & 31.5 & 4.6 & 23.1 & \\
\hline our M-WDPM-deep & 38.2 & 38.4 & 17.5 & 15.8 & 9.5 & 38.1 & 39.4 & 32.0 & 3.5 & 26.4 & \\
\hline our M-WDPM-rescore & 46.6 & 40.1 & 18.5 & 18.1 & 10.7 & 38.9 & 43.7 & 38.9 & 10.8 & 30.1 & \\
\hline Model Drift [9] & 13.4 & 44.0 & 3.1 & 3.1 & 0.0 & 31.2 & 43.9 & 7.1 & 0.1 & 9.3 & \\
\hline Multi-fold MIL [10] & 35.8 & 40.6 & 8.1 & 7.6 & 3.1 & 35.9 & 41.8 & 16.8 & 1.4 & 23.0 & \\
\hline Min-Supervision [11] & 27.6 & 41.9 & 19.7 & 9.1 & 10.4 & 35.8 & 39.1 & 33.6 & 0.6 & 20.9 & \\
\hline Pattern Config [42] & 36.3 & 47.6 & 23.3 & 12.3 & 11.1 & 36.0 & 46.6 & 25.4 & 0.7 & 23.5 & \\
\hline Posterior Reg. [43] & 42.2 & 43.9 & 23.1 & 9.2 & 12.5 & 44.9 & 45.1 & 24.9 & 8.3 & 24.0 & \\
\hline Convex Clustering [20] & 46.2 & 46.9 & 24.1 & 16.4 & 12.2 & 42.2 & 47.1 & 35.2 & 7.8 & 28.3 & \\
\hline LCL-pLSA [19] & 48.8 & 41.0 & 23.6 & 12.1 & 11.1 & 42.7 & 40.9 & 35.5 & 11.1 & 36.6 & \\
\hline DPM $5.0^{\dagger}[2]$ & 33.2 & 60.3 & 10.2 & 16.1 & 27.3 & 54.3 & 58.2 & 23.0 & 20.0 & 24.1 & \\
\hline DP-DPM conv $5^{\dagger}[36]$ & 42.3 & 65.1 & 32.2 & 24.4 & 36.7 & 56.8 & 55.7 & 38.0 & 28.2 & 47.3 & \\
\hline $\mathrm{R}_{-} \mathrm{CNN}^{\dagger}[4]$ & 68.1 & 72.8 & 56.8 & 43.0 & 36.8 & 66.3 & 74.2 & 67.6 & 34.4 & 63.5 & \\
\hline method / class & table & $\overline{\mathrm{dog}}$ & horse & mbike & person & plant & sheep & $\overline{\text { sofa }}$ & train & $\overline{\mathrm{tv}}$ & mean \\
\hline our M-WDPM-HOG & 9.4 & 24.2 & 29.8 & 42.5 & 9.1 & 14.5 & 18.3 & 11.2 & 32.1 & 14.3 & 22.6 \\
\hline our M-WDPM-deep & 11.2 & 26.1 & 33.1 & 43.7 & 8.8 & 16.7 & 20.8 & 14.5 & 33.5 & 18.0 & 24.3 \\
\hline our M-WDPM-rescore & 16.3 & 26.9 & 37.4 & 42.1 & 12.9 & 18.9 & 22.5 & 16.2 & 38.1 & 19.6 & 27.4 \\
\hline Model Drift [9] & 9.9 & 1.5 & 29.4 & 38.3 & 4.6 & 0.1 & 0.4 & 3.8 & 34.2 & 0 & 13.9 \\
\hline Multi-fold MIL [10] & 4.9 & 14.1 & 31.9 & 41.9 & 19.3 & 11.1 & 27.6 & 12.1 & 31.0 & 40.6 & 22.4 \\
\hline Min-Supervision [11] & 10.0 & 27.7 & 29.4 & 39.2 & 9.1 & 19.3 & 20.5 & 17.1 & 35.6 & 7.1 & 22.7 \\
\hline Pattern Config [42] & 12.5 & 23.5 & 27.9 & 40.9 & 14.8 & 19.2 & 24.2 & 17.1 & 37.7 & 11.6 & 24.6 \\
\hline Posterior Reg. [43] & 13.9 & 18.6 & 31.6 & 43.6 & 7.6 & 20.9 & 26.6 & 20.6 & 35.9 & 29.6 & 26.4 \\
\hline Convex Clustering [20] & 12.7 & 21.5 & 30.1 & 42.4 & 7.8 & 20.0 & 26.8 & 20.8 & 35.8 & 29.6 & 27.7 \\
\hline LCL-pLSA [19] & 18.4 & 35.3 & 34.8 & 51.3 & 17.2 & 17.4 & 26.8 & 32.8 & 35.1 & 45.6 & 30.9 \\
\hline DPM $5.0^{\dagger}[2]$ & 26.7 & 12.7 & 58.1 & 48.2 & 43.2 & 12.0 & 21.1 & 36.1 & 46.0 & 43.5 & 33.7 \\
\hline DP-DPM conv $5^{\dagger}[36]$ & 37.1 & 39.2 & 61.0 & 56.4 & 52.2 & 26.6 & 47.0 & 35.0 & 51.2 & 56.1 & 44.4 \\
\hline $\mathrm{R}_{-\mathrm{CNN}}^{\dagger}{ }^{\dagger}[4]$ & 54.5 & 61.2 & 69.1 & 68.6 & 58.7 & 33.4 & 62.9 & 51.1 & 62.5 & 64.8 & 58.5 \\
\hline
\end{tabular}

to represent the discovered object windows, its performance $(22.7 \%)$ is more or less the same with our HOG based M-WDPM, which proves the stronger discrimination of our windows selection method. When using the deep features with additional training data from ImageNet [34], our M-WDPMdeep can achieve an mAP of $24.3 \%$. The boost $(1.7 \%)$ is not as much as that of the annotation task (4\%, see Section. III-B3), it is probably because the use of distinct measuring criteria (mean average precision v.s percent of correct localization). Our detection rescoring method M-WDPM-rescore continues to improve the average precision ( $\mathrm{mAP}=27.4 \%$ ) for nearly all the classes except for the motorbike class. It shows a better performance when compared with [42], [43], and it has a competitive performance when compared with [20]. The performance gap (3.5\%) between ours and that of [19] might be partly caused by the use different deep feature representations as discussed in III-B3. We achieve the best detection results for the boat, cat, horse and train classes for this dataset.

In addition, we provide the results obtained by popular supervised object detection methods [2], [36], [4] in the bottom lines of Table IV. One can see that there is still a gap between the weakly supervised detection framework and supervised ones, although our weakly supervised DPM yields better results for some classes (e.g., aeroplane, bird, cat, dog, etc.) to the supervised DPM 5.0 [2].

5) Error analysis: We present an analysis of the types of errors that our M-WDPMs make on the PASCAL VOC 2007 test set in Fig. 8. We use the diagnosis tool of [48] and consider four types of false positive (FP) errors: Loc (poor localizations), Sim (confusion with similar objects), Oth (confusion with other objects, e.g., correctly localize an object but classifying it to a wrong class) and BG (confusion with background or unlabeled objects). Cor indicates correctly located true positives (TP). We visually show the fraction of correct detections (TP) and errors of each kind (FP) among the top ranking $T$ windows in Fig. 8, where $T$ is the number of ground-truth object windows in the test set of PASCAL VOC 2007.

We consider the M-WDPM-HOG as our baseline and show the distribution of TP and each kind of FP in Fig. 8(a). We can see that the majority errors are due to poor localizations (Loc) and confusion with background regions (BG). When adopting the deep features, our M-WDPM-deep encounters less Loc and Oth, but still it suffers from the Sim and BG error (as shown in Fig. 8(b)). In contrast, after detection rescoring, our best performing method M-WDPM-rescore has less error caused by Loc, BG and Oth (Fig. 8(c)), which validates that our rescoring 

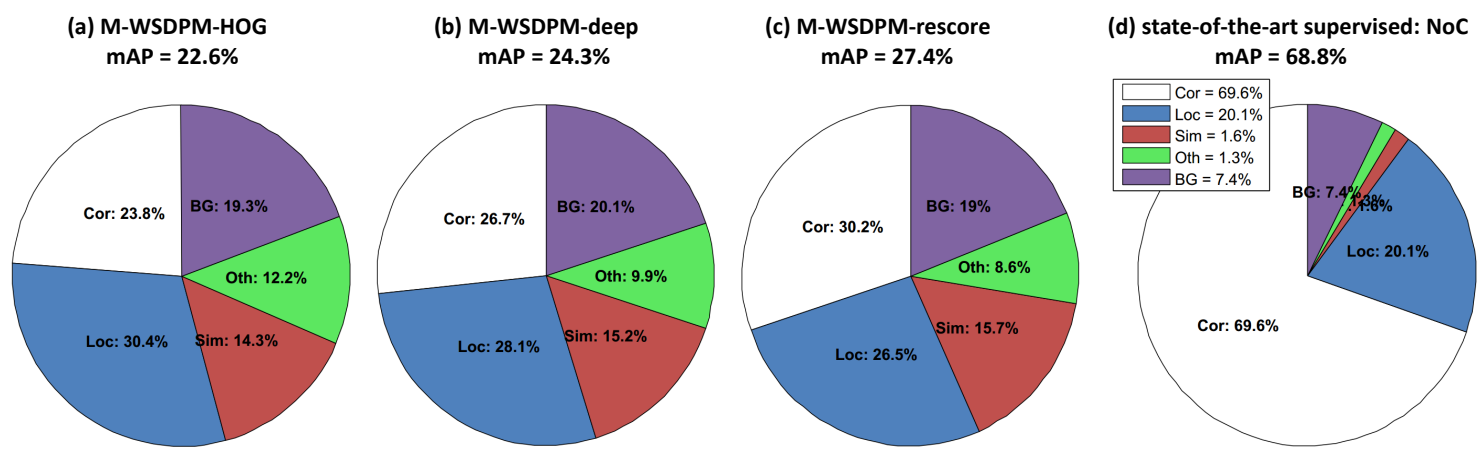

Fig. 8. Analysis of top-ranked detections on PASCAL VOC 2007 test set. Pie charts show the distributions of true positive (TP) and false positives (FP) generated by the detection error analysis tool of [48]. Percentage of top $T$ detections ( $T$ is the number of whole objects the test dataset) that are correct (Cor), or false positives due to poor localization (Loc), confusion with similar objects (Sim), confusion with other objects (Oth), or confusion with background or unlabeled objects (BG) [48]. The left three charts show the analysis of our methods, the right one is the analysis of the state-of-the-art supervised detection results obtained by NoC [49].

approach is very efficient in excluding the background regions and avoiding the misclassification. Fig. 8(d) shows the error distribution of the state-of-the-art supervised object detection framework NoC (Networks on Convolutional feature maps) [49]. NoC adopt even deeper VGG-16 [50] nets with bounding box fine-tuning on PASCAL VOC $2007+2012$ trainval. The comparison between NoC and our M-WDPM indicates that: (1) deeper network helps increasing the Cor substantially; (2) fine-tuning and supervised training with ground-truth bounding boxes yield far less Sim and Oth errors.

\section{CONCLUSION}

In this paper, we proposed a model enhancing the weakly supervised learning by emphasizing the importance of location and size of the initial class specific root filter of deformable part-based models. We follow the general setup of [17] and introduce several substantial improvements to the weakly supervised DPM. The main contributions included a new selection model based on generic "objectnes" (region proposals) and visual saliency to adaptively select a reliable set of candidate windows which tend to represent the object instances in the image, and a latent class learning process by coarsely classifying a candidate window into either target object or non-target class. Furthermore we designed a flexible enlarging-and-shrinking post-processing procedure to modify the output bounding boxes of DPM, which can effectively further improve the final accuracy. Experimental results on the challenging PASCAL VOC 2007 database according to various criteria demonstrate that our proposed framework is efficient and competitive with the state-of-the-arts.

\section{REFERENCES}

[1] N. Dalal and B. Triggs, "Histograms of oriented gradients for human detection," in CVPR, 2005.

[2] P. F. Felzenszwalb, R. B. Girshick, D. McAllester, and D. Ramanan, "Object detection with discriminatively trained part based models," IEEE TPAMI, vol. 32, no. 9, pp. 1627-1645, 2010.

[3] C. Szegedy, A. Toshev, and D. Erhan, "Deep neural networks for object detection," in NIPS, 2013.

[4] R. Girshick, J. Donahue, T. Darrell, and J. Malik, "Rich feature hierarchies for accurate object detection and semantic segmentation," in $C V P R, 2014$.
[5] O. Maron and A. L. Ratan, "Multiple-instance learning for natural scene classification," in ICML, 1998.

[6] S. Andrews, I. Tsochantaridis, and T. Hofmann, "Support vector machines for multiple-instance learning," in NIPS, 2003.

[7] C. Galleguillos, B. Babenko, A. Rabinovich, and S. Belongie, "Weakly supervised object recognition and localization with stable segmentation$\mathrm{s}$, , in $E C C V, 2008$.

[8] M. Nguyen, L. Torresani, F. de la Torre, and C. Rother, "Weakly supervised discriminative localization and classification: a joint learning process," in ICCV, 2009.

[9] P. Siva and T. Xiang, "Weakly supervised object detector learning with model drift detection," in ICCV, 2011.

[10] R. Cinbis, J. Verbeek, and C. Schmid, "Multi-fold mil training for weakly supervised object localization," in CVPR, 2014.

[11] H. O. Song, R. Girshick, S. Jegelka, J. Mairal, Z. Harchaoui, and T. Darrell, "On learning to localize objects with minimal supervision," in ICML, 2014

[12] T. Deselaers, B. Alexe, and V. Ferrari, "Weakly supervised localization and learning with generic knowledge," IJCV, vol. 100, no. 3, pp. 275293, 2012.

[13] J. D. Lafferty, A. McCallum, and F. C. N. Pereira, "Conditional random fields: Probabilistic models for segmenting and labeling sequence data," in $I C M L, 2001$.

[14] B. Alexe, T. Deselaers, and V. Ferrari, "Measuring the objectness of image windows," IEEE TPAMI, vol. 34, no. 11, pp. 2189-2202, 2012.

[15] Z. Shi, P. Siva, and T. Xiang, "Transfer learning by ranking for weakly supervised object annotation," in BMVC, 2012.

[16] J. Hoffman, S. Guadarrama, E. Tzeng, R. Hu, J. Donahue, R. Girshick, T. Darrell, and K. Saenko, "LSDA: Large scale detection through adaptation," in NIPS, 2014.

[17] M. Pandey and S. Lazebnik, "Scene recognition and weakly supervised object localization with deformable part-based models," in ICCV, 2011.

[18] Z. Shi, T. M. Hospedales, and T. Xiang, "Bayesian joint topic modelling for weakly supervised object localisation," in ICCV, 2013.

[19] C. Wang, K. Huang, W. Ren, J. Zhang, and S. Maybank, "Large-scale weakly supervised object localization via latent category learning," IEEE $T I P$, vol. 24, no. 4, pp. 1371-1385, April 2015.

[20] H. Bilen, M. Pedersoli, and T. Tuytelaars, "Weakly supervised object detection with convex clustering," in CVPR, 2015.

[21] R. Girshick, P. Felzenszwalb, and D. McAllester, "Object detection with grammar models," in NIPS, 2011.

[22] H. Azizpour and I. Laptev, "Object detection using strongly-supervised deformable part models," in ECCV, 2012.

[23] X. Ren and D. Ramanan, "Histograms of sparse codes for object detection," in CVPR, 2013.

[24] M. Everingham, L. Van Gool, C. K. I. Williams, J. Winn, and A. Zisserman, "The pascal visual object classes (voc) challenge," IJCV, vol. 88, no. $2,2010$.

[25] J. Uijlings, K. van de Sande, T. Gevers, and A. Smeulders, "Selective search for object recognition," IJCV, 2013.

[26] Y. Tang, X. Wang, E. Dellandrea, S. Masnou, and L. Chen, "Fusing generic objectness and deformable part-based models for weakly supervised object detection," in ICIP, 2014. 
[27] M.-M. Cheng, Z. Zhang, W.-Y. Lin, and P. Torr, "Bing: Binarized normed gradients for objectness estimation at 300fps," in CVPR, 2014

[28] C. L. Zitnick and P. Dollár, "Edge boxes: Locating object proposals from edges," in $E C C V, 2014$

[29] J. Carreira and C. Sminchisescu, "Cpmc: Automatic object segmentation using constrained parametric min-cuts," IEEE TPAMI, vol. 34, no. 7, pp. 1312-1328, July 2012.

[30] P. Arbeláez, J. Pont-Tuset, J. Barron, F. Marques, and J. Malik, "Multiscale combinatorial grouping," in CVPR, 2014.

[31] J. Hosang, R. Benenson, and B. Schiele, "How good are detection proposals, really?" in BMVC, 2014.

[32] A. Krizhevsky, I. Sutskever, and G. E. Hinton, "Imagenet classification with deep convolutional neural networks," in NIPS, 2012.

[33] Y. Jia, E. Shelhamer, J. Donahue, S. Karayev, J. Long, R. Girshick, S. Guadarrama, and T. Darrell, "Caffe: Convolutional architecture for fast feature embedding," arXiv preprint arXiv:1408.5093, 2014.

[34] O. Russakovsky, J. Deng, H. Su, J. Krause, S. Satheesh, S. Ma, Z. Huang, A. Karpathy, A. Khosla, M. Bernstein, A. C. Berg, and L. Fei-Fei, "Imagenet large scale visual recognition challenge," arXiv: $1409.0575,2014$.

[35] C.-C. Chang and C.-J. Lin, "Libsvm: A library for support vector machines," ACM Trans. Intell. Syst. Technol., vol. 2, no. 3, pp. 27:127:27, May 2011.

[36] R. Girshick, F. Iandola, T. Darrell, and J. Malik, "Deformable part models are convolutional neural networks," in CVPR, 2015

[37] P. Sermanet, D. Eigen, X. Zhang, M. Mathieu, R. Fergus, and Y. LeCun, "Overfeat: Integrated recognition, localization and detection using convolutional networks," in ICLR 2014, 2014.

[38] K. He, X. Zhang, S. Ren, and J. Sun, "Spatial pyramid pooling in deep convolutional networks for visual recognition," IEEE TPAMI, vol. PP, no. 99, pp. 1-1, 2015.

[39] X. T. Y. Ke and F. Jing, "The design of high-level features for photo quality assessment," in CVPR, 2006.

[40] P. Siva, C. Russell, and T. Xiang, "In defence of negative mining for annotating weakly labelled data," in ECCV, 2012.

[41] S. Andrews, I. Tsochantaridis, and T. Hofmann, "Support vector machines for multiple-instance learning," in NIPS, 2003.

[42] H. O. Song, Y. J. Lee, S. Jegelka, and T. Darrell, "Weakly-supervised discovery of visual pattern configurations," in NIPS, 2014.

[43] H. Bilen, M. Pedersoli, and T. Tuytelaars, "Weakly supervised object detection with posterior regularization," in BMVC, 2014.

[44] X. Zhou, K. Yu, T. Zhang, and T. S. Huang, "Image classification using super-vector coding of local image descriptors," in ECCV, 2010.

[45] D. G. Lowe, "Object recognition from local scale-invariant features," in ICCV, 1999.

[46] G. Csurka, C. Dance, L. Fan, J. Willamowski, and C. Bray, "Visual categorization with bags of keypoints," in Workshop on statistical learning in computer vision, ECCV, 2004.

[47] F. Perronnin, J. Sánchez, and T. Mensink, "Improving the fisher kernel for large-scale image classification," in ECCV, 2010.

[48] D. Hoiem, Y. Chodpathumwan, and Q. Dai, "Diagnosing error in object detectors," in ECCV, 2012.

[49] S. Ren, K. He, R. Girshick, X. Zhang, and J. Sun, "Object detection networks on convolutional feature maps," arXiv preprint arXiv:1504.06066, 2015.

[50] K. Simonyan and A. Zisserman, "Very deep convolutional networks for large-scale image recognition," in ICLR, 2015.

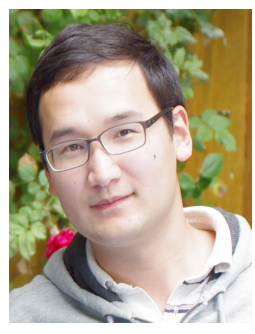

Yuxing Tang received the B.S. and M.S. degrees from the Department of Information and Telecommunication Engineering, Beijing Jiaotong University, Beijing, China, in 2009 and 2011. He is currently pursuing a Ph.D. degree with the Department of Mathematics and Computer Science, Ecole Centrale de Lyon, France. His research interests are computer vision and machine learning; in particular models for visual category recognition and object detection.

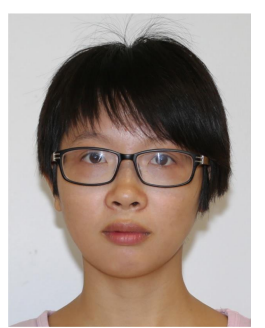

Xiaofang Wang received the B.S. and M.S. degrees in biomedical engineering from Central South University, Changsha, China. She was awarded the $\mathrm{Ph} . \mathrm{D}$. degree in Computer Science from Ecole Centrale de Lyon, France in 2015. Her current research interests include image/video processing, medical image segmentation and analysis, multiple object tracking, and semantic segmentation.

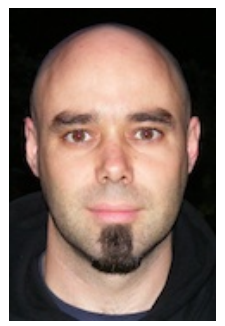

Emmanuel Dellandréa was awarded his Master and Engineering degrees in Computer Science from the University of Tours, France, in 2000 followed by his Ph.D. in Computer Science in 2003. He then joined the Ecole Centrale de Lyon, France, in 2004 as an Associate Professor. His research interests include multimedia analysis, image and audio understanding and affective computing, including recognition of affect from image, audio and video signals.

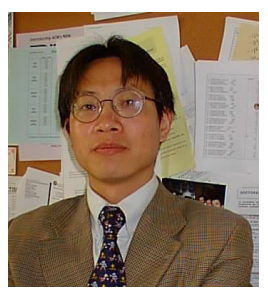

Liming Chen was awarded a joint B.Sc. degree in mathematics and computer science from the University of Nantes, France in 1984. He obtained a M.Sc. degree in 1986 and a Ph.D. degree in computer science from the University of Paris 6 in 1989. He first served as associate professor at the Universite de Technologie de Compiègne, then joined Ecole Centrale de Lyon as Professor in 1998, where he leads an advanced research team on multimedia computing and pattern recognition. From 2001 to 2003, he also served as Chief Scientific Officer in a Paris-based company, Avivias, specialized in media asset management. In 2005, he served as scientific multimedia expert in France Telecom R\&D China. He has been head of the department of mathematics and computer science from 2007.

Prof. Liming Chen has taken out three patents, authored more than 100 publications and acted as chairman, PC member and reviewer in a number of high profile journal and conferences since 1995. He has been a (co)principal investigator on a number of research grants from EU FP program, French research funding bodies and local government departments. He has directed more than $30 \mathrm{Ph} . \mathrm{D}$. theses. His current research spans from 2D/3D face analysis and recognition, image and video analysis and categorization, to affect analysis in image, audio and video. 